\title{
Vasculogenic mimicry is associated with increased tumor-infiltrating neutrophil and poor outcome in esophageal squamous cell carcinoma
}

This article was published in the following Dove Press journal:

OncoTargets and Therapy

9 June 2017

Number of times this article has been viewed

\author{
Jingxin Zhangl,* \\ Guoxia Zhang ${ }^{2, *}$ \\ Pingping $\mathrm{Hu}^{3}$ \\ Guodong Deng ${ }^{4}$ \\ Qiqi Liu ${ }^{4}$ \\ Lili Qiao ${ }^{4}$ \\ Hui Luo' \\ Jiandong Zhang ${ }^{3}$ \\ 'Department of Oncology, \\ Weifang Medical College, Weifang, \\ 2Department of Pathology, \\ ${ }^{3}$ Department of Radiation Oncology, \\ Qianfoshan Hospital Affiliated to \\ Shandong University, ${ }^{4}$ Department of \\ Oncology, Shandong University School \\ of Medicine, Jinan, China \\ *These authors contributed equally \\ to this work
}

Correspondence: Jiandong Zhang Department of Radiation Oncology, Qianfoshan Hospital Affiliated to Shandong University, 16766 Jingshi Road, Jinan 2500I4, China

Tel +86 I3583I23486

Email zjxblt007@sina.com
Purpose: Vasculogenic mimicry (VM) is known to be a mechanism to nourish the tumor, but little is known about its prognostic significance in esophageal squamous cell carcinoma (ESCC). We characterized the predictive relevance of $\mathrm{VM}$ expression and tumor-infiltrating neutrophil (TIN) density in patients with resectable ESCC.

Methods: We retrospectively collected clinicopathologic characteristics of 117 esophageal cancer (EC) patients undergoing complete resection and without preoperative therapy. Immunohistochemistry was used to detect the expression of E-cadherin and CD66b. CD34/periodic acid-schiff (PAS) double staining was used to detect the expression of VM.

Results: VM expression was observed in $56(47.9 \%)$ patients. VM was negatively correlated with E-cadherin (correlation coefficient $=-0.364, P<0.001$ ) and was positively correlated with infiltration of CD66b neutrophil (correlation coefficient $=0.421, P<0.001$ ). $\mathrm{VM}$ and $\mathrm{CD}_{66} \mathrm{~b}^{+}$ neutrophil infiltration are important markers for poor overall survival and disease-free survival. Multivariate analysis showed that VM, $\mathrm{CD}_{6} 6 \mathrm{~b}^{+}$neutrophil infiltration, pathologic tumor node metastasis (TNM) (pTNM) stage, and tumor differentiation are significant independent prognostic predictors in ECs $(P=0.001,0.025,0.001,0.011$, respectively). VM expression is identified in $\sim 47.9 \%$ of ESCC, and it is associated with poor outcome and increased TIN.

Conclusion: TIN is an important factor for VM formation. Therefore, studies of invasive ability of EC in patients with VM could supply significant information for therapeutic strategy.

Keywords: E-cadherin, CD66b, cancer, CD34/PAS double staining, prognosis

\section{Background}

Esophageal cancer (EC) is a common malignant tumor and is one of high-risk tumors in China. Early metastasis and postoperative recurrence are the main causes of the high mortality in EC. ${ }^{1}$ The mortality of EC has not been dramatically improved despite the decreased incidence and improved management. ${ }^{2}$ Novel insights into underlying molecular oncogenic mechanisms are needed for development of better therapies.

"Vasculogenic mimicry" (VM), which was first reported in melanoma in 1999, refers to tumor cells directly interconnected to form vessel-like channels to transport blood $^{3}$ and was found in many other malignant tumors, including ovarian cancer, breast cancer, prostate cancer, osteosarcoma, bladder cancer, colorectal cancer, gastric cancer, lung cancer, and hepatocellular cancer. ${ }^{4} \mathrm{VM}$ plays an important role in nourishing the tumor, forming an extracellular matrix (ECM)-rich network, taking tumor cells with blood directly into the microcirculation environment and metastasizing to other organs, and thus playing a significant role in tumor growth and metastasis. ${ }^{5}$ However, 
little is known about the immune microenvironment of VM. Among the cells that comprise the immune microenvironment, polymorphonuclear neutrophils (PMNs) have recently been shown to play pivotal roles by altering the immune responses, which are also correlated with patient survival in various cancers. ${ }^{6,7}$ Indeed, VM and tumor-infiltrating neutrophil (TIN) have been demonstrated to be prognostic factors in various tumors. ${ }^{8-10}$ But no study has evaluated the association between them in esophageal squamous cell carcinoma (ESCC).

TIN can induce epithelial-mesenchymal transition (EMT) of tumor cells and facilitate the transition from epithelial cells into dedifferentiated mesenchymal state, allowing the loss of contact inhibition, the remodeling of ECM, and promoting a more invasive phenotype. ${ }^{11-13}$ Furthermore, strong evidence exists between EMT and VM. It is shown that EMT-related factors are upregulated in VM-forming tumor cells. ${ }^{14}$

The clinical relevance of VM and TIN in resectable ESCC has remained unclear. Based on the previous views above, we hypothesized that VM might be associated with TIN by the crucial bridge of EMT. In the present study, we examined VM, EMT biomarker, and TIN density in ESCC patients and investigated their prognosis value and predictive relevance.

\section{Methods}

\section{Patients and specimens}

From December 2007 to May 2013, 117 patients with primary ESCC, undergoing complete resection and without preoperative therapy, were enrolled in our study from Qianfoshan Hospital Affiliated to Shandong University. Patients who died within 30 days of surgery or with positive margins were excluded. The nontumor death patients should also be ruled out. This research was approved by the Ethical Committee of Qianfoshan Hospital Affiliated to Shandong University, and written informed consent was obtained from all individual participants included in the study. The clinical information was collected from retrieving the medical records including sex, age, smoking status, tobacco history, alcohol consumption, adjuvant therapy, pathologic tumor node metastasis (pTNM) stage, lymph node metastasis, and the greatest dimension of primary tumor. Laboratory data including white blood cell (WBC) count, neutrophilic granulocyte (NEU) count, lymphocyte (LYM) count, and monocyte (MON) count were collected. The first end point was overall survival (OS) defined as the time from operation to the date of death, the date lost to follow-up, or the date of the latest follow-up. The second end point was disease-free survival (DFS), defined as the time from operation to the first recurrence or death.

\section{Immunohistochemical staining}

Specimens, sectioned from archived, paraffin-embedded tumor tissues, were cut into $4-\mu \mathrm{m}$ serial sections. Then, the sections were dewaxed twice in xylene and rehydrated by ethanol. Subsequently, heat-mediated antigen retrieval was performed by applying sodium citrate buffer $(\mathrm{pH} 6.0)$ at $100^{\circ} \mathrm{C}$ for 2 minutes. Endogenous peroxidase was removed with $3 \% \mathrm{H}_{2} \mathrm{O}_{2}$. Monoclonal rabbit antihuman CD34 antibody (1:200 dilution, ab81289; Abcam, Cambridge, UK), monoclonal rabbit antihuman E-cadherin (1:300 dilution; CST; 3195S), and the primary antibody of TIN (CD66b, 1:50 dilution, ab197678; Abcam) were added onto the sections and incubated overnight. Then, the sections were washed with PBS and incubated with goat antirabbit biotinylated secondary antibody (SP-9001; Zhongshan Biotechnology, Beijing, China) for 15 minutes at $37^{\circ} \mathrm{C}$, followed by horseradish peroxidase (HRP)-conjuncted streptavidin (SP-9001; Zhongshan Biotechnology) incubation at $37^{\circ} \mathrm{C}$ for 30 minutes. Diamino-benzidine (DAB) was used to detect the immunoactivity. Sections were counterstained with hematoxylin. Finally, sections were dehydrated with ethanol gradient and mounted by rhamsan gum.

\section{CD34/PAS double staining}

CD34 immunohistochemical staining was completed, and the sections were incubated with periodic acid solution for 7 minutes and then washed in distilled water for 3 minutes. Schiff solution was used to the sections for 20 minutes. After distilled water rinsing, hematoxylin staining and gradient dehydration were applied prior to mounting with neutral gum.

\section{Scoring of immunohistochemistry}

Two independent pathologists analyzed all the immunohistochemistry (IHC) results blindly. The degree of E-cadherin expression was scored by a semiquantitative method. Classifying the intensity of staining was used by a range of $0-3$ : negative, 0 point; weak, 1 point; moderate, 2 points; strong, 3 points. The percentage of positive cells stained was recorded according to the following four grade: positive cells $<10 \%$, 0 points; $10 \%-40 \%$ positive cells, 1 point; $40 \%-70 \%$ positive cells, 2 points; $\geq 70 \%$ positive cells, 3 points. The product of these two scores was used to estimate the results..$^{15}$ In this study, all the final scores were stratified as low expression (0-6 scores) and high expression (7-12 scores). 
The criterion quantitating immunohistochemical staining of TIN was the number of neutrophils in tumor nest, assessing in 10 nonoverlapping high-power fields (HPFS; magnification of $\times 400 ; 0.08 \mathrm{~mm}^{2}$ ) with representative $\mathrm{CD} 6 \mathrm{~b}^{+}$staining. The median value was obtained for further evaluation.

\section{VM judgment}

VM could be seen to be formed by tumor cells without endothelial cells. CD34/PAS double-staining was used to validate VM. The vascular endothelium was identified by positive CD34 staining. Lumens with CD34-negative/PASpositive, as wells as walls composed of tumor cells, was verified by hematoxylin-eosin staining (HE staining). The above cases were then divided into two groups: VM-positive and VM-negative groups.

\section{Statistical analysis}

Data analyses were performed by using SPSS software program (version 17.0; SPSS Inc., IL, USA). Correlation of VM with clinicopathological features was evaluated by using Pearson's $\chi^{2}$ or Fisher's exact test. The correlations among VM, E-cadherin, and CD66b were analyzed by Spearman's rho analyses. Univariate and multivariate COX analyses were performed to determine the relation between VM and clinical characteristics and to analyze whether VM was an independent prognostic factor for ESCC patients. $P<0.05$ was considered statistically significant.

\section{Results}

\section{Identification of and relation between VM, E-cadherin, and CD66b}

Total population included 117 patients, comprising 17 males and 100 females. The median age was 63 (range, 46 to 80). Of these, $84(71.8 \%)$ patients were regarded as stage I $\sim$ II and
$33(28.2 \%)$ as stage III depending on pTNM stage. There were $83(71 \%)$ cases of well and moderate differentiation and $34(29 \%)$ cases of poor differentiation.

Positive VM was observed in 56 (47.9\%) patients based on the CD34/PAS double staining, which was characterized by PAS-positive loops surrounding with tumor cells, with or without red blood cells in it, meanwhile having no CD34 staining (Figure 1A). The expression levels of E-cadherin and CD66b were tested by IHC in the corresponding specimens (Figure 1B and $\mathrm{C}$ ). E-cadherin expression on $\mathrm{EC}$ is extensive ( $>85 \%$ in the examined specimens). But high expression of E-cadherin (score $\geq 6$ ) was tested only in 35 $(29.9 \%)$ patients. Low expression of E-cadherin (score <6) was observed in $82(70.1 \%)$ patients. We found that most of the positive-VM specimens expressed low level of E-cadherin, and the negative-VM specimens expressed high level of E-cadherin. The results demonstrated that VM was negatively correlated with E-cadherin (correlation coefficient $=-0.364, P<0.001$, Figure $2 \mathrm{~A}$ and B). Moreover, VM was significantly positively correlated with the infiltration of $\mathrm{CD} 6 \mathrm{~b}^{+}$neutrophil $(400 \times$, Figure $2 \mathrm{C}$ and D) in the same sample (correlation coefficient $=0.421, P<0.001$ ). E-cadherin was negatively associated with the infiltration of $\mathrm{CD}_{66 \mathrm{~b}^{+}}$neutrophil $(400 \times$, Figure $2 \mathrm{E}$ and $\mathrm{F})$ in the same specimen (correlation coefficient $=-0.468, P<0.001$ ).

\section{Clinical significance of $\mathrm{VM}$ in EC}

Our analysis demonstrated that VM was significantly correlated with pTNM stage $(P=0.003)$, E-cadherin $(P<0.001)$, and TIN $\left(\mathrm{CD}_{66 \mathrm{~b}}\right)^{+}(P<0.001)$ expression. Whereas no statistically significant association was shown between VM and sex, age, tobacco smoking, alcohol drinking, tumor differentiation, lymph node metastasis, WBC, NEU, LYM, and MON (Tables 1 and 2).
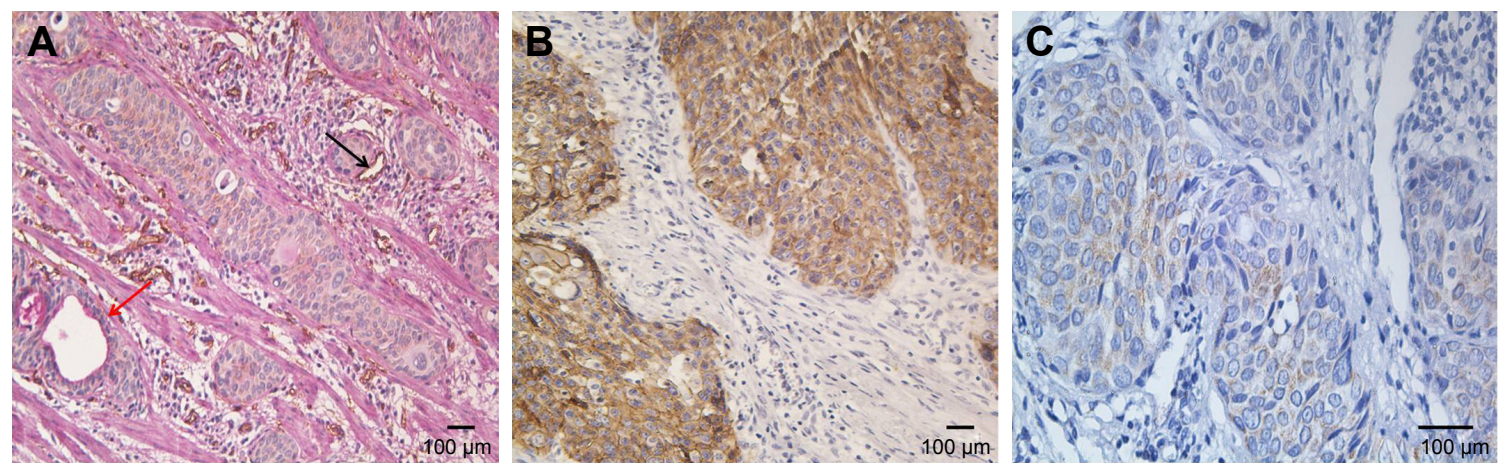

Figure I IHC of paraffin-embedded esophageal cancer. (A) Hematoxylin-eosin staining of VM channel on the left lined only by tumor cells which are negative for CD34 staining (red arrow). The panel on the right shows endothelium-dependent vessels are positive for CD34 staining (black arrow) (magnification: $200 \times$ ). (B) E-cadherin expression in esophageal cancer (magnification: 200x). (C) CD66b expression in esophageal cancer (magnification: 400×).

Abbreviations: IHC, immunohistochemistry; VM, vasculogenic mimicry. 

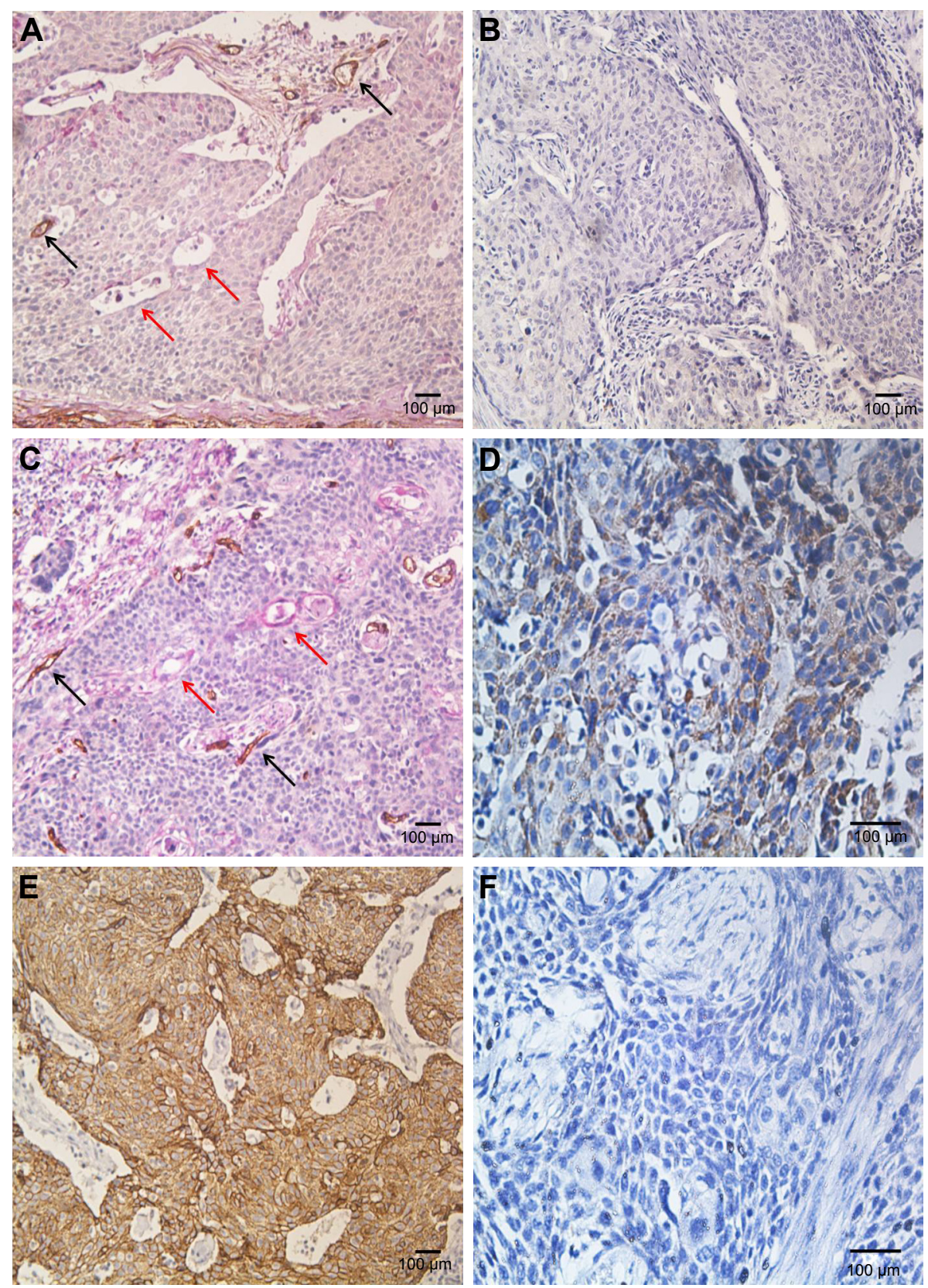

Figure 2 The expressions of VM (A and $\mathbf{C})$, E-cadherin (B and $\mathbf{E})$, and tumor-infiltrating neutrophil (CD66b, D and $\mathbf{F})$ in esophageal cancer. (A, 200x) The expression of VM (red arrows and black arrows refer to endothelium-dependent vessels) was positive, whereas the expression of E-cadherin was weak in the same sample (B, 200×). (C, 200X) The expression was VM was existent, and the expression of tumor-infiltrating neutrophil was strong in the same sample (D, 400x). (E, 200x) The expression of E-cadherin was strong, whereas tumor-infiltrating neutrophil was almost absent in the same sample (F, 400x).

Abbreviation: VM, vasculogenic mimicry.

\section{Survival analysis}

Median follow-up was 36 months, including $73.5 \%$ of patients with over 24 months, $53 \%$ over 36 months, and $12 \%$ over 60 months. Progression was observed in 117 patients in the whole cohort, with 61 (52.1\%) patients in negative-VM group, 56 (47.9\%) patients in positive-VM group, 82 (70.1\%) patients in E-cadherin low-expression group, 35 (29.9\%) patients in E-cadherin in high-expression group, 46 (39.3\%) patients in CD66b low-expression group, and 71 (60.7\%) patients in high-expression group. A total of 77 patients died at the last follow-up. We evaluated the prognostic value of VM, E-cadherin, and CD66b related to two points: OS and DFS. Kaplan-Meier curves of the six patient groups above for OS and DFS are displayed in Figure 3A-F, respectively. The survival is significantly different between the positive-VM group and negative-VM group (OS, log-rank 
Table I Clinicopathologic significance of VM in II7 esophageal squamous cell carcinoma patients

\begin{tabular}{|c|c|c|c|c|}
\hline Variables & $\begin{array}{l}\text { Number of } \\
\text { patients (\%) }\end{array}$ & VM (-) & VM (+) & $P$-value \\
\hline Overall number & 117 & 61 & 56 & \\
\hline Sex & & & & $0.099^{a}$ \\
\hline Female & $17(14.5)$ & 12 & 5 & \\
\hline Male & $100(85.5)$ & 49 & 51 & \\
\hline Age (years) & & & & $0.117^{a}$ \\
\hline$<65$ & $59(50.4)$ & 35 & 24 & \\
\hline$\geq 65$ & $58(49.6)$ & 26 & 32 & \\
\hline Tobacco smoking & & & & $0.409^{\mathrm{a}}$ \\
\hline Yes & $85(72.6)$ & 42 & 43 & \\
\hline No & $31(27.4)$ & 18 & 13 & \\
\hline Alcohol drinking & & & & $0.565^{\mathrm{a}}$ \\
\hline Yes & $87(74.4)$ & 44 & 43 & \\
\hline No & $30(25.6)$ & 17 & 13 & \\
\hline \multicolumn{3}{|c|}{ Degree of tumor differentiation } & & $0.482^{\mathrm{a}}$ \\
\hline I (poor) & $34(29)$ & 16 & 18 & \\
\hline II III (moderate $\sim$ well) & $83(7 I)$ & 45 & 38 & \\
\hline \multicolumn{3}{|c|}{ Tumor node metastasis (TNM) stage } & & $0.003^{\mathrm{a}}$ \\
\hline $\mid \sim \|$ & $84(71.8)$ & 51 & 33 & \\
\hline III & $33(28.2)$ & 10 & 23 & \\
\hline Lymph node metastasis & & & & $0.183^{\mathrm{a}}$ \\
\hline Yes & 49 & 22 & 27 & \\
\hline No & 68 & 39 & 29 & \\
\hline
\end{tabular}

Note: aP-value for Pearson's $\chi^{2}$.

Abbreviation: VM, vasculogenic mimicry.

test, $\chi^{2}=28,762, P<0.001$; DFS, log-rank test, $\chi^{2}=25.320$, $P<0.001$ ), and statistically significant differences were observed between CD66b low- and high-expression groups (OS, log-rank test, $\chi^{2}=10.962, P<0.001$; DFS, log-rank test, $\left.\chi^{2}=9.225, P=0.002\right)$. But there is no statistically significant difference between E-cadherin low-expression group and high-expression group (OS, log-rank test, $\chi^{2}=0.051$, $P=0.821 ; \mathrm{DFS}, \log$-rank test, $\left.\chi^{2}=0.064, P=0.800\right)$. Therefore,

Table 2 Spearman's rho analyses of VM and clinicopathological features

\begin{tabular}{lll}
\hline Clinicopathological & $\begin{array}{l}\text { Correlation } \\
\text { coefficient }\end{array}$ & P-value \\
\hline Age & 0.126 & 0.177 \\
TNM stage & 0.274 & 0.003 \\
Differentiation & -0.065 & 0.486 \\
Lymph node metastasis & -0.123 & 0.186 \\
Tumor dimension & 0.075 & 0.420 \\
WBC & -0.076 & 0.417 \\
NEU & -0.064 & 0.492 \\
LYM & -0.069 & 0.468 \\
MON & -0.170 & 0.067 \\
E-cadherin expression & -0.364 & 0.000 \\
TIN (CD66b) expression & 0.421 & 0.000 \\
\hline
\end{tabular}

Abbreviations: TNM, tumor node metastasis; WBC, white blood cell; NEU, neutrophil; LYM, lymphocyte; MON, monocyte; TIN, tumor-infiltrating neutrophil; VM, vasculogenic mimicry. it is demonstrated that VM and the infiltration of $\mathrm{CD}^{2} 6 \mathrm{~b}^{+}$ neutrophil are important markers for poor prognosis. In univariate Cox analysis, the expressions of VM, pTNM stage, lymph node metastasis, degree of tumor differentiation, tumor dimension, and TIN (CD66b) were all statistically significant predictors for OS $(P<0.001,0.001,0.038$, $0.031,0.028,0.001$, respectively). Our multivariate analysis, in which variables were tested in a step-forward logistic regression approach, showed that VM was proved to be an independent prognostic predictor (hazard rate $[\mathrm{HR}]=0.275$; 95\% confidence interval $[\mathrm{CI}]=0.163 \sim 0.401462 ; P<0.001)$; meanwhile, the degree of tumor differentiation, pTNM stage, and TIN (CD66b) expression are also independent prognostic predictors $(\mathrm{HR}=2.018,95 \% \mathrm{CI}=1.218 \sim 3.344$, $P=0.006 ;$ HR $=0.234,95 \%$ CI $=0.105 \sim 0.521, P<0.001$; $\mathrm{HR}=0.487,95 \% \mathrm{CI}=0.259 \sim 0.916, P=0.025$, respectively) (Table 3). Statistically significant outcomes of the study analysis demonstrated that positive VM could be used as a potential marker for prognosis.

\section{Discussion}

It is well known that blood supply is a key factor for tumor growth, invasion, and metastasis. Currently, there are three patterns providing blood supply for tumors: VM, mosaic vessels, and endothelium-dependent vessels. ${ }^{16}$ Among the three patterns, VM plays crucial roles in providing blood supply for tumors. Different from angiogenesis and vasculogenesis being the widely accepted mechanisms, VM refers to pipes surrounded by the tumor cells but without depending on vessel endothelial cells. It is an important way to develop tumor invasion and metastasis. ${ }^{17} \mathrm{VM}$ had been found to exist in esophageal stromal tumors. ${ }^{18}$ Thus far, few studies have examined VM in ESCC. This study demonstrated for the first time that VM was associated with patients' outcome in resectable ESCC and discovered that OS and DFS of VM-positive patients are shorter than those of VM-negative patients.

Many research studies have investigated the clinical implications of VM and TIN..$^{12,19-24}$ Although some of their biologic roles have been revealed, the relations among VM and TIN in the context of EC have not yet been established. In a previous study, Hu et al demonstrated that TIN could contribute to EMT in lung adenocarinoma cells and esophageal squamous cancer cell., ${ }^{9,25}$ TIN was revealed to be beneficial for tumor growth, invasion, and metastasis by proteolysis of ECM components, promoting angiogenesis and mediating immunosuppression. ${ }^{26}$ TIN was an independent predictor of unfavorable outcome in intrahepatic cholangiocarcinoma, 
A
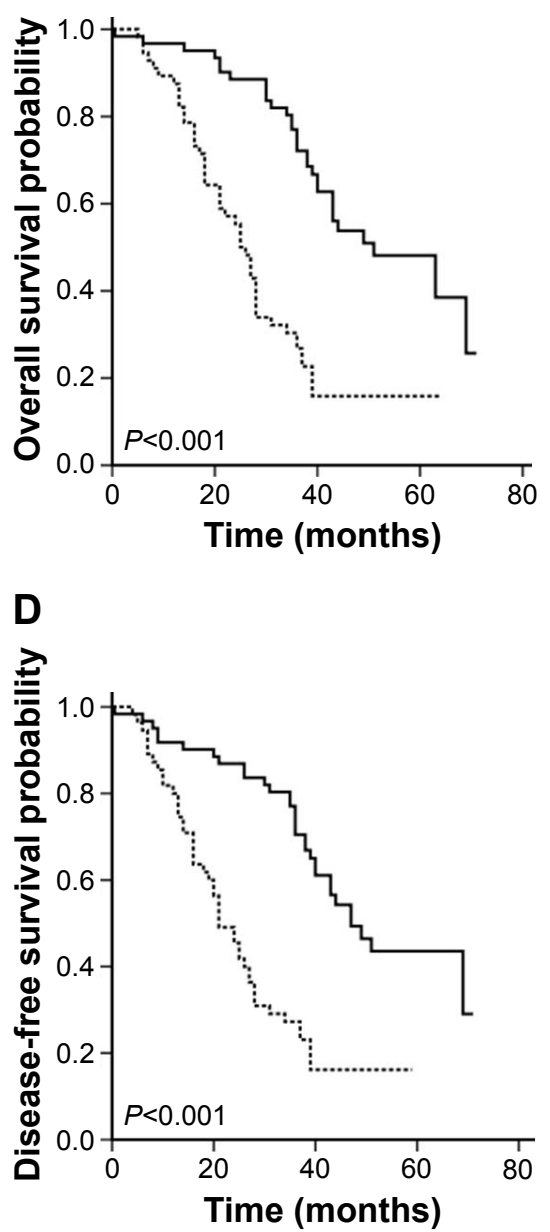

$\square$ VM (-)
...$:$ VM (+)
B

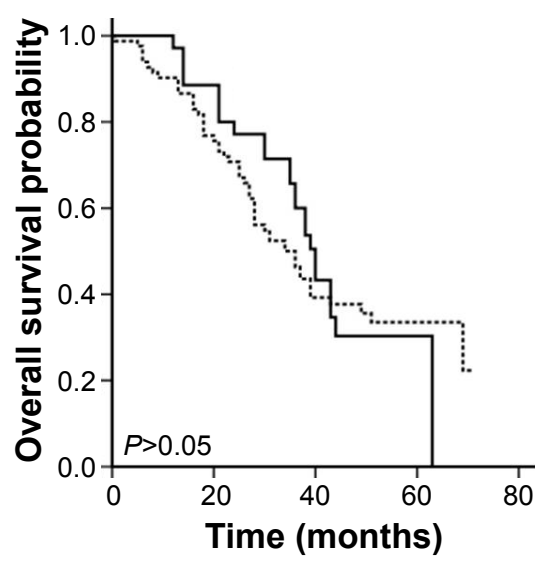

E

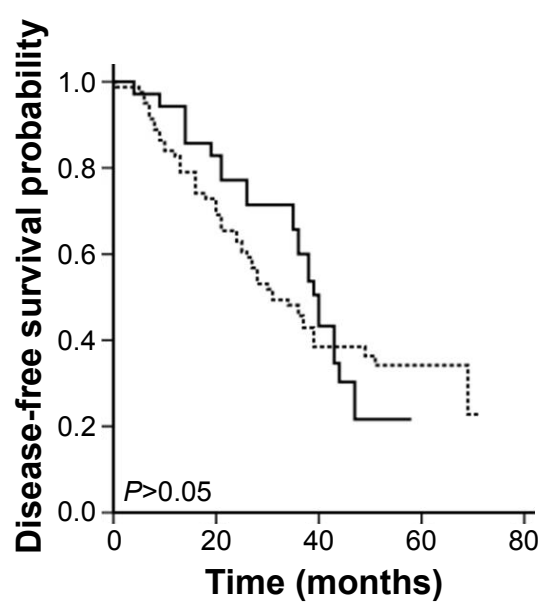

..:- E-cadherin low expression $\neg$ E-cadherin high expression
C
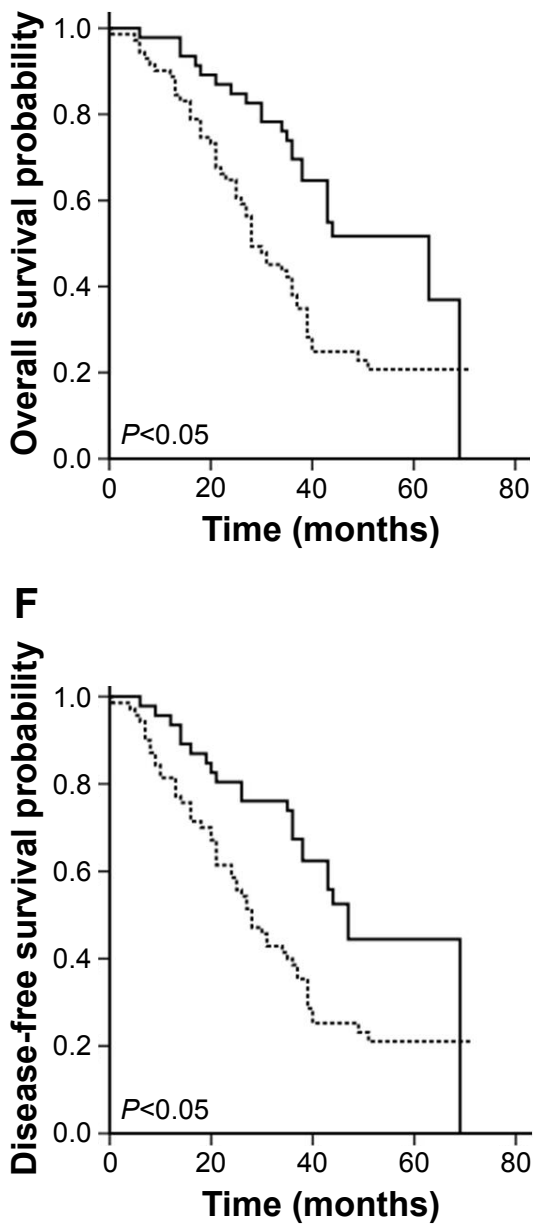

$\neg$ CD66b low expression ..*: CD66b high expression

Figure 3 Kaplan-Meier curves for clinical reference values. It is significantly different between positive-VM group and negative-VM group (A: OS, log-rank test, $\chi^{2}=28,762$, $P<0.00$ I; D: DFS, log-rank test, $\chi^{2}=25.320, P<0.00 \mathrm{I}$ ), and statistically significant differences were found between CD66b low-expression group and CD66b high-expression group (C: OS, log-rank test, $\chi^{2}=10.962, P=0.001 ; \mathrm{F}$ : DFS, log-rank test, $\chi^{2}=9.225, P=0.002$ ). But there is no statistically significant difference between $\mathrm{E}$-cadherin lowexpression group and high-expression group (B: OS, log-rank test, $\chi^{2}=0.05 \mathrm{I}, P=0.82 \mathrm{I}$; E: DFS, log-rank test, $\chi^{2}=0.064, P=0.800$ ).

Abbreviations: VM, vasculogenic mimicry, OS, overall survival; DFS, disease-free survival.

hepatocellular cancer, head and neck cancer, renal cell cancer, non-small cell lung cancer, and EC.., 26

The biologic determinants of the relation between VM and TIN are not well understood. EMT is widely acknowledged to be involved in cancer invasion and metastasis. ${ }^{3}$ Further evidences have found that EMT regulators and EMT-related transcription factors are highly upregulated in VM-forming tumor cells. ${ }^{27}$ Many studies have demonstrated that adhesion molecules and other factors modulating EMT process can regulate VM formation, such as Twist, ZEB1, Snail, and Slug/Snail2. ${ }^{14}$ In this study, we performed an IHC stain of E-cadherin, a well-established indicator of EMT, and discovered that VM was associated with EMT. But EMT is not related to a poor outcome in EC patients. Based on the evidences above, we suspected whether TIN was related to VM? In our study, we performed an IHC stain of CD66b and concluded that TIN was related to poor outcome in EC patients. The result of the analysis of correlation between VM and TIN expression revealed that VM was correlated with TIN. To our knowledge, this is the first study investigate the relation between VM and TIN through IHC. The correlation between VM and TIN leads to the presumption that TIN could induce VM by the interaction of TIN and tumor cells in a tumor microenvironment. According to the correlation between EMT and TIN in a previous study, and the relation between VM and EMT, we speculated that TIN might develop VM formation through inducing EMT progression in tumor microenvironment. Recruitment of TIN to the tumor microenvironment could induce EMT in EC cells, and then promote VM, which 
Table 3 Cox-proportional hazard analyses

\begin{tabular}{|c|c|c|c|c|}
\hline \multirow[t]{2}{*}{ Overall survival } & \multicolumn{2}{|l|}{ Univariate } & \multicolumn{2}{|l|}{ Multivariate } \\
\hline & HR (95\% Cl) & $P$-value & HR $(95 \% \mathrm{Cl})$ & $P$-value \\
\hline Sex & & 0.747 & & \\
\hline Male & $0.903(0.487 \sim 0.747)$ & & & \\
\hline Female & I & & & \\
\hline Age (years) & & 0.082 & & \\
\hline$<65$ & $0.668(0.424 \sim 1.052)$ & & & \\
\hline$\geq 65$ & I & & & \\
\hline pTNM stage & & $<0.001$ & & $<0.001$ \\
\hline $\mid \sim \|$ & $0.296(0.183 \sim 0.473)$ & & $0.176(0.076 \sim 0.409)$ & \\
\hline III & I & & I & \\
\hline Lymph node metastasis & & 0.038 & & \\
\hline Yes & $1.610(1.027 \sim 2.524)$ & & & \\
\hline No & I & & & \\
\hline Degree of tumor & & 0.031 & & 0.011 \\
\hline \multicolumn{5}{|l|}{ differentiation } \\
\hline I (poor) & $1.703(1.064 \sim 2.724)$ & & $1.943(1.163 \sim 3.249)$ & \\
\hline II III (moderate $\sim$ well) & I & & I & \\
\hline Tumor dimension & & 0.028 & & \\
\hline$d<5$ & $0.602(0.382 \sim 0.948)$ & & & \\
\hline$d \geq 5$ & I & & & \\
\hline TIN (CD66b) expression & & 0.001 & & 0.025 \\
\hline Low expression & $0.450(0.276 \sim 0.736)$ & & $0.487(0.259 \sim 0.916)$ & \\
\hline High expression & I & & I & \\
\hline VM & & $<0.001$ & & 0.001 \\
\hline VM $(-)$ & $0.297(0.185 \sim 0.477)$ & & $0.369(0.207 \sim 0.658)$ & \\
\hline VM (+) & I & & I & \\
\hline
\end{tabular}

Abbreviations: $\mathrm{HR}$, hazard rate; $\mathrm{Cl}$, confidence interval; TIN, tumor-infiltrating neutrophil; VM, vasculogenic mimicry; PTNM, pathologic tumor node metastasis.

resulted in dismal outcome. Further studies are required to clarify this hypothesis.

In our study, pTNM stage, lymph node metastasis, tumor differentiation, and tumor dimension were all the important features and critical prognostic factors of EC. But the expression of VM does not relate intimately with them above. Presentation and function of VM are decided by several characteristics, factors, and microenvironments during tumor growth. Our team tended to the view that microenvironment is the greatest effect for VM. Qiao et al have published a review about the VM. ${ }^{4}$ In the review, the mechanisms of VM formation were listed clearly, such as MMP2, Ln-5, VE-cadherin, EphA2, and hypoxia. Few evidence could prove that VM is associated with pTNM stage, lymph node metastasis, tumor differentiation, and tumor dimension in EC. Moreover, the number of samples collected was not enough to verify the correlation above. In a further study, we will use a large sample to elucidate the correlation.

Although we could not provide definitive mechanisms in this finding because of the lack of experimental evidence, these indicators may be useful for stratifying patients in clinical settings. More importantly, our findings may also contribute to establishing a basis for the potential mechanism of VM and tumor immunity for ESCC.

In conclusion, this is the first study to determine the prognostic value of VM in EC patients. It highlights VM as an independent predictor of poor OS and DFS in EC. It is the first time to explore the relation among VM, EMT, and TIN. TIN is an important factor for VM formation. Therefore, studies of invasive ability of EC in patients with VM could supply significant information for therapeutic strategy. Such information may help medical scientists predicting patients' outcome early and provide a reasonable individualized treatment.

\section{Ethics approval}

All procedures performed in this study were in accordance with the ethical standards of the institutional research committee and with the 1964 Helsinki Declaration and its later amendments or comparable ethical standards.

\section{Informed consent}

Written informed consent was obtained from all individual participants included in the study. 


\section{Acknowledgments}

This thesis would not have been possible without the contributions of several people. To all these people, we wish to express our gratitude. We would like to acknowledge and extend our heartfelt gratitude to Jian Xie, Ning Liang, Fangjie Chen, Wei Jiang, and Ning Li for their vital encouragement and patient guidance, generous assistance, and invaluable advice, all of which have been of inestimable worth to the completion of our thesis. This study was supported by grants from the National Natural Science Foundation of China (grant no 81672974 and no 81602719).

\section{Disclosure}

The authors report no conflicts of interest in this work.

\section{References}

1. Siegel RL, Miller KD, Jemal A. Cancer statistics, 2016. CA Cancer J Clin. 2016;66(1):7-30.

2. Chen W, Zheng R, Baade PD, et al. Cancer statistics in China, 2015. CA Cancer J Clin. 2016;66(2):115-132.

3. Maniotis AJ, Folberg R, Hess A, et al. Vascular channel formation by human melanoma cells in vivo and in vitro: vasculogenic mimicry. Am J Pathol. 1999;155(3):739-752.

4. Qiao L, Liang N, Zhang J, et al. Advanced research on vasculogenic mimicry in cancer. J Cell Mol Med. 2015;19(2):315-326.

5. Sun T, Zhao N, Zhao XL, et al. Expression and functional significance of Twist 1 in hepatocellular carcinoma: its role in vasculogenic mimicry. Hepatology. 2010;51(2):545-556.

6. Nathan C. Neutrophils and immunity: challenges and opportunities. Nat Rev Immunol. 2006;6(3):173-182.

7. Donskov F. Immunomonitoring and prognostic relevance of neutrophils in clinical trials. Semin Cancer Biol. 2013;23(3):200-207.

8. Caruso RA, Bellocco R, Pagano M, Bertoli G, Rigoli L, Inferrera C. Prognostic value of intratumoral neutrophils in advanced gastric carcinoma in a high-risk area in Northern Italy. Mod Pathol. 2002;15(8): 831-837.

9. Wang J, Jia Y, Wang N, et al. The clinical significance of tumorinfiltrating neutrophils and neutrophil-to-CD8+ lymphocyte ratio in patients with resectable esophageal squamous cell carcinoma. J Transl Med. 2014;12:7.

10. Zhao JJ PK, Wang W, Chen JG, et al. The prognostic value of tumorinfiltrating neutrophils in gastric adenocarcinoma after resection. PLoS One. 2012;7(3):e33655.
11. Fan YL, Zheng M, Tang YL, Liang XH. A new perspective of vasculogenic mimicry: EMT and cancer stem cells (Review). Oncol Lett. 2013;6(5):1174-1180.

12. Wang W, Lin P, Han C, Cai W, Zhao X, Sun B. Vasculogenic mimicry contributes to lymph node metastasis of laryngeal squamous cell carcinoma. J Exp Clin Cancer Res. 2010;29:60.

13. Thiery JP, Acloque H, Huang RY, Nieto MA. Epithelial-mesenchymal transitions in development and disease. Cell. 2009;139(5):871-890.

14. Liu Q, Qiao L, Liang N, et al. The relationship between vasculogenic mimicry and epithelial-mesenchymal transitions. J Cell Mol Med. 2016;20(9):1761-1769.

15. Dass SD CP, Ong DB, Teoh KH, Looi LM. E-cadherin downregulation at the infiltrating tumour front is associated with histological grade and stage in colorectal carcinoma of Malaysians. Malays J Pathol. 2015; 37(1):19-24.

16. Folberg R, Hendrix MJ, Maniotis AJ. Vasculogenic mimicry and tumor angiogenesis. Am J Pathol. 2000;156(2):361-381.

17. Lin $P$, Wang W, Sun BC, et al. Vasculogenic mimicry is a key prognostic factor for laryngeal squamous cell carcinoma: a new pattern of blood supply. Chin Med J. 2012;125(19):3445-3449.

18. Zhao H, Gu XM. Study on vasculogenic mimicry in malignant esophageal stromal tumors. World J Gastroenterology. 2008;14(15):2430-2433.

19. Liang J, Yang B, Cao Q, Wu X. Association of vasculogenic mimicry formation and CD133 expression with poor prognosis in ovarian cancer. Gynecol Obstet Invest. 2016;81(6):529-536.

20. Zhang Y, Sun B, Zhao X, et al. Clinical significances and prognostic value of cancer stem-like cells markers and vasculogenic mimicry in renal cell carcinoma. J Surg Oncol. 2013;108(6):414-419.

21. Chen L, He Y, Sun S, Sun B, Tang X. Vasculogenic mimicry is a major feature and novel predictor of poor prognosis in patients with orbital rhabdomyosarcoma. Oncol Lett. 2015;10(3):1635-1641.

22. Donskov F. Impact of immune parameters on long-term survival in metastatic renal cell carcinoma. J Clin Oncol. 2006;24(13):1997-2005.

23. Rao HL CJ, Li M, et al. Increased intratumoral neutrophil in colorectal carcinomas correlates closely with malignant phenotype and predicts patients' adverse prognosis. PLoS One. 2012;7(1):e30806.

24. Jensen TO, Schmidt H, Møller HJ, et al. Intratumoral neutrophils and plasmacytoid dendritic cells indicate poor prognosis and are associated with pSTAT3 expression in AJCC stage I/II melanoma. Cancer. 2012;118(9):2476-2485.

25. Hu P, Shen M, Zhang P, et al. Intratumoral neutrophil granulocytes contribute to epithelial-mesenchymal transition in lung adenocarcinoma cells. Tumour Biol. 2015;36(10):7789-7796.

26. Shen M, Hu P, Donskov F, Wang G, Liu Q, Du J. Tumor-associated neutrophils as a new prognostic factor in cancer: a systematic review and meta-analysis. PLoS One. 2014;9(6):e98259.

27. Na DC, Lee JE, Yoo JE, Oh BK, Choi GH, Park YN. Invasion and EMTassociated genes are upregulated in B viral hepatocellular carcinoma with high expression of CD133-human and cell culture study. Exp Mol Pathol. 2011;90(1):66-73.
OncoTargets and Therapy

\section{Publish your work in this journal}

OncoTargets and Therapy is an international, peer-reviewed, open access journal focusing on the pathological basis of all cancers, potential targets for therapy and treatment protocols employed to improve the management of cancer patients. The journal also focuses on the impact of management programs and new therapeutic agents and protocols on

\section{Dovepress}

patient perspectives such as quality of life, adherence and satisfaction. The manuscript management system is completely online and includes a very quick and fair peer-review system, which is all easy to use. Visit http://www.dovepress.com/testimonials.php to read real quotes from published authors. 